\title{
Reconciling turbulent burning velocity with flame surface area in small-scale turbulence
}

\author{
G. V. Nivarti, R. S. Cant, and S. Hochgreb \\ University Engineering Department, Cambridge - United Kingdom
}

(Received xx; revised xx; accepted xx)

A discrepancy between the enhancement in overall burning rate and the enhancement in flame surface area measured for high-intensity turbulence is addressed. In order to reconcile the two quantities, an additional contribution from the effective turbulent diffusivity is considered. This contribution is expected to arise in sufficiently intense turbulence from eddies smaller than the flamelet thickness. In the present work, the enhancement in diffusivity arising from these eddies is estimated based on a model energy spectrum; individual contributions from all turbulence length scales smaller the flamelet thickness are integrated over the corresponding portion of the spectrum. It is shown that diffusivity enhancement, estimated in this manner, is able to account for the measured discrepancy between the overall burning rate enhancement and flame surface area enhancement. The factor quantifying this discrepancy is formalized as a closed-form function of the Karlovitz number.

Key words: Turbulent burning velocity, High-intensity turbulence, Thin Reaction Zones regime, Damköhler's hypotheses, Flamelet modelling.

\section{Introduction: burning velocity enhancement in turbulence}

\subsection{Corrugated Flamelets Regime and Damköhler's first hypothesis}

Flamelet modelling of turbulent flame propagation relies on the first of two hypotheses laid out by Damköhler (1940). According to this hypothesis (DH1), the overall burning rate $\dot{m}$ quantified by the turbulent burning velocity $s_{T}$ is linearly proportional to the turbulent surface area $A_{T}$ of the propagating flame. This relationship is expressed as

$$
\frac{s_{T}}{s_{L}} \sim \frac{A_{T}}{A}=\left(1+\frac{\Delta A}{A}\right),
$$

where $s_{L}$ is the laminar burning velocity corresponding to the unburned mixture, and $A$ is the mean unwrinkled flame surface area. The expression on the right indicates that the enhancement in $s_{T}$ with respect to $s_{L}$ is related to the enhancement $\Delta A=A_{T}-A$ of turbulent flame surface area beyond the unwrinkled area $A$, as determined by the isosurface of a suitable reaction progress variable.

DH1 strictly applies only when all turbulence length scales $\ell$ are much larger than the laminar flame thickness $\delta_{L}$. In that case, the smallest (Kolmogorov) length scale $\eta$ satisfies $\eta>\delta_{L}$. This regime of turbulent flame propagation - referred to as the Corrugated Flamelets (CF) regime (Peters 1999) - corresponds to a value less than unity 
of the Karlovitz number Ka defined (Bray \& Cant 1991) as

$$
\mathrm{Ka} \equiv \frac{\delta_{L}^{2}}{\eta^{2}}=\left(\frac{u^{\prime}}{s_{L}}\right)^{3 / 2}\left(\frac{\delta_{L}}{\ell_{0}}\right)^{1 / 2},
$$

where the relation on the right expresses the definition in terms of the laminar burning velocity $s_{L}$, the laminar flame thickness, the integral length scale $\ell_{0}$, and the turbulence intensity $u^{\prime}$ following Peters (1999). This equality relies on the assumption that the kinematic viscosity $\nu \sim s_{L} \delta_{L}$ and the Reynolds number $\operatorname{Re} \equiv u^{\prime} \ell_{0} / \delta_{L} \sim\left(\ell_{0} / \eta\right)^{4 / 3}$. Experiments have indicated persistently (Bradley 1992; Driscoll 2008) that DH1 remains valid in the $\mathrm{CF}$ regime, i.e. for $\mathrm{Ka}<1$ or as long as $\eta>\delta_{L}$. The question is whether DH1 remains valid in the small-scale turbulence regime with $\eta<\delta_{L}$, for which Ka $>1$.

In recent years, two separate experimental investigations - conducted in Toronto (Yuen \& Gülder 2013) and in Michigan (Wabel et al. 2017) - have disclosed a common result for a wide range of high Ka values. In these experiments, turbulent Bunsen flames are stabilized by an annular pilot methane-air flame or ethylene-air flame (Toronto) or by a co-flow of hot products produced by the same methane-air mixture (Michigan). The turbulent burning velocity $s_{T}$ is calculated from the reactant inflow rate $\dot{m}$, assuming that the entire incoming fuel-air mixture is burned across the flame so that $\dot{m}=\rho A s_{T}$, where $\rho$ is the density of the fuel-air mixture. Hence, the relative accuracy of resulting $s_{T} / s_{L}$ measurements is equal to the accuracy of $\dot{m}$ measurements which is within $5 \%$ of the stated values (Yuen 2009; Wabel 2017). The total flame surface area $A_{T}$ is measured using an appropriate isosurface of a marker: temperature (Toronto) or $\mathrm{OH}$ radical (Michigan), which is visualized using laser induced Rayleigh scattering (Toronto) or Laser Induced Fluorescence (Michigan). The total flame surface area $A_{T}$ is calculated using the method of Shepherd (1996): the flame surface density is determined from the marker isosurface and subsequently integrated over the flame brush which is assumed to possess cylindrical symmetry. The accuracy of the resulting $A_{T} / A$ measurements is related to errors arising from the pixel resolution as well as those arising from off-plane flame surface normals. In the Toronto measurements, the total error in flame surface area measurements is estimated to be about $15-20 \%$ (Yuen 2009). In the Michigan measurements, the former is estimated to be up to $3 \%$ and the latter is estimated to be about 18\% (Wabel 2017). In general, the error arising from off-plane flame surface normals are difficult to quantify. However, a recent Direct Numerical Simulation (DNS) study of turbulent jet flames (Wang et al. 2016) has shown that the multiplicative correction factor needed on a 2D flame surface area measurement tends to be unity near the inlet and approaches the value $4 / \pi \approx 1.27$ in the downstream region (as derived by Veynante et al. $(2010)$ ). The uncertainty in the Karlovitz numbers is related to the measurement of $u^{\prime} / s_{L}$ and $\ell_{0} / \delta_{L}$. In both experiments, these quantities were measured at the base of the flame using Laser Doppler Anemometry, with uncertainties of $1-3 \%$, leading to uncertainties in the Karlovitz number Ka of at most $4 \%$.

The discrepancy between the profiles of $s_{T} / s_{L}$ and $A_{T} / A$ measured at Toronto and Michigan is shown in Fig. 1 by plotting the ratio $\left(s_{T} / s_{L}\right) /\left(A_{T} / A\right)$ as a function of Ka. For low values of $\mathrm{Ka}$, the enhancement $s_{T} / s_{L}$ is entirely accounted for by the flame surface area enhancement $A_{T} / A$, as per DH1. In other words, the ratio $\left(s_{T} / s_{L}\right) /\left(A_{T} / A\right)$ is close to unity for $\mathrm{Ka} \sim 1$. For higher values of $\mathrm{Ka}$, the turbulent burning velocity enhancement $s_{T} / s_{L}$ and the enhancement in flame surface area $A_{T} / A$ show an increasing disparity, i.e. $\left(s_{T} / s_{L}\right) /\left(A_{T} / A\right)>1$ for $\mathrm{Ka} \gg 1$. The Michigan experiments (blue circles in Fig. 1), which have examined a wider range of Ka values, indicate a disparity of up to one order of magnitude for $\mathrm{Ka} \sim 10^{3}$. At any given value of $\mathrm{Ka}$, the experimental 


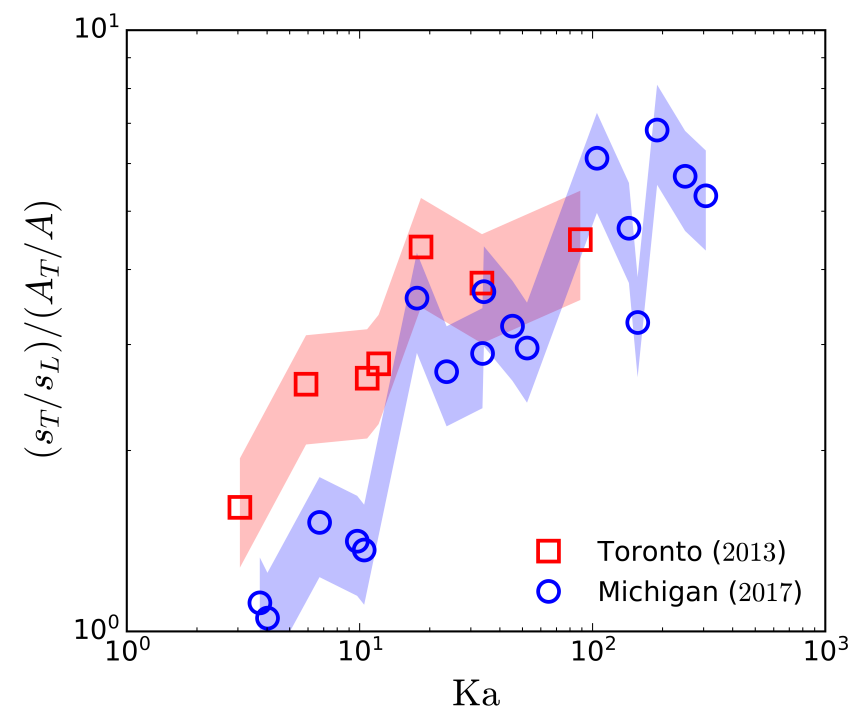

Figure 1. Measured disparity in the profiles of turbulent burning velocity enhancement $s_{T} / s_{L}$ and turbulent surface area enhancement $A_{T} / A$ obtained by the Toronto (Yuen \& Gülder 2013) and Michigan (Wabel et al. 2017) experiments. Shaded regions in red and blue denote error bars for the Toronto and Michigan experiments, respectively.

results from Toronto (red squares in Fig. 1) yield marginally higher disparities, yet the general behaviour observed is the same for both experiments: $A_{T} / A$ fails to account for the overall enhancement $s_{T} / s_{L}$ in turbulent burning velocity for $\mathrm{Ka}>1$.

Why do the profiles of $s_{T} / s_{L}$ and $A_{T} / A$ diverge for $\mathrm{Ka}>1$ ? What other mechanism, if not the area enhancement according to DH1, could possibly account for the enhancement $s_{T} / s_{L}$ over and above the enhancement $A_{T} / A$ ? These open questions are addressed in the present work. In both experiments (Yuen \& Gülder 2013; Wabel et al. 2017), a thickening effect is referred to at high Ka values, and the flame surface marker interface appears to get smeared with increasing Ka. This observation provides a clue towards the interpretation of a likely additional mechanism of $s_{T}$ enhancement as follows.

\subsection{Thin Reaction Zones Regime and Damköhler's second hypothesis}

In small-scale turbulence with $\mathrm{Ka}>1$, the action of length scales $\ell<\delta_{L}$ may be considered. These scales may provide a further contribution to the enhancement of $s_{T}$ over and above the area enhancement $A_{T} / A$. The second of Damköhler's two hypotheses (Damköhler 1940) presents a mechanism of $s_{T}$ enhancement by turbulence length scales smaller than $\delta_{L}$. According to Damköhler's second hypothesis (DH2), the overall burning velocity enhancement $s_{T} / s_{L}$ in small-scale turbulence varies as a function of the enhancement $D_{T} / D$ in the effective turbulent diffusivity $D_{T}$. This is expressed as

$$
\frac{s_{T}}{s_{L}}=\sqrt{\frac{D_{T}}{D}}=\left(1+\frac{\Delta D}{D}\right)^{1 / 2},
$$

where $D$ is the molecular diffusivity in laminar flow. This dependence is an extension to turbulent flames of the dependence formulated for the laminar burning velocity by Zel'dovich \& Frank-Kamenetskii (1938). As written previously for DH1 (Eq. (1.1)), the expression on the right shows that the turbulent burning velocity enhancement $s_{T} / s_{L}$ 


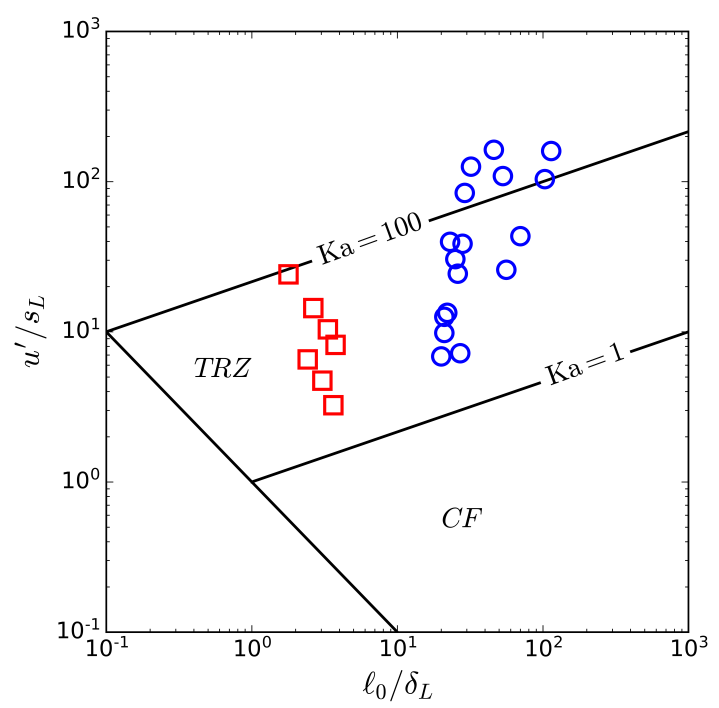

FiguRE 2. Turbulent flame propagation regimes in the Toronto (red squares) and Michigan (blue circles) experiments.

is related to the turbulent diffusivity enhancement $\Delta D=D_{T}-D$ over and above the molecular diffusivity $D$.

Damköhler (1940) had originally envisioned DH2 to apply strictly to turbulence with all length scales $\ell$ much smaller than the laminar flame thickness $\delta_{L}$. This implies that the largest (integral) length scale satisfies $\ell_{0}<\delta_{L}$ in the regime corresponding to DH2. Such a regime, however, is seldom obtained in practice because the integral length scale $\ell_{0}$ of an experimentally-generated turbulent flow is typically several times greater than the laminar flame thickness $\delta_{L}$ of the unburned mixture (Driscoll 2008). Hence, an intermediate regime between those corresponding to DH1 and DH2 is more relevant to practical conditions. In this intermediate regime, termed the Thin Reaction Zones (TRZ) regime (Peters 1999), some turbulence length scales are smaller than $\delta_{L}$, while the remaining length scales are larger than $\delta_{L}$. Hence, for the TRZ regime, the integral length scale $\ell_{0}>\delta_{L}$ and the Kolmogorov scale $\eta<\delta_{L}$, and the Karlovitz number satisfies $\mathrm{Ka}>1$. Fig. 2 shows the regimes corresponding to the Toronto and Michigan experiments (Yuen \& Gülder 2013; Wabel et al. 2017) on a Borghi diagram. It is evident that most experiments correspond to the TRZ regime with $\mathrm{Ka}>1$; some experiments from the Michigan data (blue circles in Fig. 2) even correspond to $\mathrm{Ka}>100$. The additional mechanism that accounts for the enhancement $\left(s_{T} / s_{L}\right) /\left(A_{T} / A\right)$ over and above the area enhancement $A_{T} / A$ must, therefore, be pertinent to the TRZ regime.

Since some turbulence length scales are smaller than the laminar flame thickness in the TRZ regime, these smaller scales may be surmised to affect the diffusive processes in the preheat zone of the flamelet (without disturbing the reaction zone). The action of these small-scales $\ell<\delta_{L}$ may be expected to be over and above the action of largescales $\ell>\delta_{L}$, which act to enhance the flame surface area (without disturbing the processes internal to the flamelet). The related enhancement of $s_{T}$ due to turbulence small-scales has been estimated (Zimont 1979) from the turbulent diffusivity expected at the scale of the broadened flame brush thickness using semi-empirical arguments. Unfortunately, no comparisons with experimental data were presented alongside. Ronney \& Yakhot (1992) applied a similar approach using the Kolmogorov energy spectrum to 
compare the enhancement in $s_{T}$ predicted for the TRZ regime with experimental data of Abdel-Gayed et al. (1987). While a 'fair' agreement with experiments was found, it was assumed the small-scales lie within the inertial sub-range, i.e. the energy spectrum and the semi-empirical formulation of enhanced diffusivity did not include a model of the dissipation range. More recently, Gülder (2007) has provided yet another estimate for the diffusive enhancement of $s_{T}$ due to turbulence small-scales by taking intermittency into account. Assuming that the small-scale diffusive effects dominate at the Taylor length scale $\lambda$, the related enhancement in diffusivity is derived as a function of the Taylor-scale Reynolds number $\operatorname{Re}_{\lambda} \equiv u^{\prime} \lambda / \nu$. Limited experimental data was addressed in this work with regard to the range of Karlovitz numbers. Following a slightly different approach, Lee \& Huh (2012) have provided expressions of $s_{T}$ enhancement spanning a range of turbulence conditions based on estimates of the mean curvature and turbulent diffusivity at the flame brush leading edge. This approach leads to a reasonable agreement with experimental data (Lee et al. 2014), but requires closure of the mean curvature term under varying turbulence conditions and tuning of the constants involved. These attempts have, therefore, left a gap for an accurate but simplified formulation that addresses the relatively recent experiments, which span a wider range of Karlovitz numbers.

Along the lines of the studies mentioned above, the present work provides a formulation for the contribution of diffusivity enhancement $D_{T} / D$ towards the burning velocity enhancement $s_{T} / s_{L}$ over and above the area enhancement $A_{T} / A$ recorded in the TRZ regime. Following this concept, the overall turbulent burning velocity enhancement $s_{T} / s_{L}$ in the Toronto and Michigan experiments (Yuen \& Gülder 2013; Wabel et al. 2017) is written as a combination of DH1 and DH2 as follows

$$
\frac{s_{T}}{s_{L}}=\underbrace{\left(1+\frac{\Delta A}{A}\right)}_{\text {large-scale }} \underbrace{\left(1+\frac{\Delta D}{D}\right)^{1 / 2}}_{\text {small-scale }}
$$

where the enhancement factor $\Delta A / A$ is attributed to turbulence large-scales $\ell>\delta_{L}$, its effect directly measurable as an increase in flame area which is adopted from the Toronto and Michigan experiments. The enhancement factor $\Delta D / D$ is attributed to turbulence small-scales $\ell<\delta_{L}$. Here, in contrast to the previous investigations (Zimont 1979; Ronney \& Yakhot 1992; Gülder 2007; Lee \& Huh 2012), the factor $\Delta D / D$ and its contribution to $s_{T} / s_{L}$ is computed straightforwardly from a model energy spectrum that includes an exponential decay of energy at high wavenumbers (Pope 2000); this model spectrum is known to agree well with non-reacting experiments, and there is evidence of agreement with turbulent reacting flow experiments (Wang et al. 2007). Further, no assumption regarding the dominant scale of action of diffusivity enhancement is adopted, unlike Zimont (1979), Ronney \& Yakhot (1992), or Gülder (2007). Rather, the total diffusive effect of all turbulence length scales $\ell$ smaller than the laminar flame thickness $\delta_{L}$ is estimated. The estimate obtained is directed towards reconciling the enhancement factors $s_{T} / s_{L}$ and $A_{T} / A$ measured in contemporary experiments (at Toronto and Michigan) which span a greater range of Karlovitz numbers than before. In line with previous studies, no model is included to account for the effect of flame quenching. The mathematical approach for estimating the enhancement factor $\Delta D / D$ is presented in Section 2. This factor is compared with the disparity between DH1 and DH2 given by $\left(s_{T} / s_{L}\right) /\left(A_{T} / A\right)$ in Section 3 ; encouraging results are obtained, and a discussion of ongoing and further work is provided in Section 4. 


\section{Theory: diffusivity enhancement in small-scale turbulence}

Prandtl's hypothesis is adopted to estimate the diffusivity enhancement arising from turbulence small-scales. Accordingly, the turbulent diffusivity $D_{\ell}$ at a length scale $\ell$ in turbulent flow is assumed to be proportional to the product of the velocity $u_{\ell}$ and the length $\ell$ of the turbulent motion, i.e. $D_{\ell}=\ell u_{\ell}$. Subsequently, it is assumed that length scales $\ell<\delta_{L}$ contribute to the diffusivity enhancement $\Delta D$.Hence, the overall diffusivity enhancement factor $\Delta D$ can be computed as the average

$$
\Delta D=\int_{\delta_{L}^{-1}}^{\infty} \frac{u(\kappa)}{\kappa} d \kappa,
$$

over the range of wavenumbers corresponding to length scales $\ell=0 \rightarrow \delta_{L}$ in the flow. This amounts to considering all wavenumbers $\kappa>\delta_{L}^{-1}$ towards the average diffusivity enhancement. (It may argued that the smallest length scale in turbulent flow is the Kolmogorov scale $\eta$, and the upper limit be taken as $\eta^{-1}$ rather than $\infty$. Due to the rapid decay of energy at high wavenumbers, either choice leads to the same functional form in the final result). A natural scheme of scaling is to use properties of the laminar flame: thickness $\delta_{L}$ and speed $s_{L}$. Thus, the molecular diffusivity $D=s_{L} \delta_{L}$, and the overall diffusivity enhancement factor $\Delta D / D$ is obtained as

$$
\frac{\Delta D}{D}=\int_{1}^{\infty} \frac{\hat{u}(\hat{\kappa})}{\hat{\kappa}} d \hat{\kappa},
$$

with the ^symbol denoting scaled variables: the scaled wavenumber $\hat{\kappa}=\kappa \delta_{L}$, and the scaled velocity $\hat{u}(\hat{\kappa})=\sqrt{2 \hat{E}(\hat{\kappa})}$ where $\hat{E}(\hat{\kappa})$ is the scaled energy spectrum. Presently, a model energy spectrum based on the Kolmogorov spectrum is adopted as presented by Pope (2000). This model includes inertial scale (von Karman 1948) and dissipation scale (Kraichnan 1959) spectra as follows

$$
\hat{E}(\hat{\kappa})=C \hat{\varepsilon}^{2 / 3} \hat{\kappa}^{-5 / 3} \hat{f}_{L}(\hat{\kappa}) \hat{f}_{\eta}(\hat{\kappa})
$$

where $C \sim 1.5$ is a parametric constant of order unity. The spectral functions $\hat{f}_{L}(\hat{\kappa})$ and $\hat{f}_{\eta}(\hat{\kappa})$ correspond to the energy spectrum profiles in the energy containing range and the dissipation range, respectively. For length scales $\ell<\delta_{L}$, the inertial range spectrum $\hat{f}_{L}(\hat{\kappa}) \rightarrow 1$ because $\hat{\kappa} L / \delta_{L} \gg 1$ in this range for a specified geometrical dimension $L$. For the dissipation range spectrum $\hat{f}_{\eta}(\hat{\kappa})$, an exponential profile is specified as follows

$$
\hat{f}_{\eta}(\hat{\kappa}) \sim e^{-\beta \hat{\kappa} \eta / \delta_{L}},
$$

where $\beta \sim 5.2$ is an empirically-obtained parametric constant (Pope 2000). Hence, the overall diffusivity enhancement factor $\Delta D / D$ is written as

$$
\frac{\Delta D}{D}=(2 C)^{1 / 2} \hat{\varepsilon}^{1 / 3} \int_{1}^{\infty} \hat{\kappa}^{-11 / 6} e^{-\beta \hat{\kappa} /(2 \sqrt{\mathrm{Ka}})} d \hat{\kappa},
$$

where the substitution $\delta_{L} / \eta=\sqrt{\mathrm{Ka}}$ has been made. The term $\hat{\varepsilon}$ is the scaled form of the turbulent kinetic energy dissipation rate $\varepsilon$, which is given by $\varepsilon=15 \nu\left(u^{\prime} / \lambda\right)^{2}$ according to Taylor (1935), where $\lambda$ is the Taylor length scale and $\nu$ is the kinematic viscosity. The scaled dissipation rate $\hat{\varepsilon}$ can be formulated as follows

$$
\begin{aligned}
\hat{\varepsilon} & =\varepsilon \frac{\delta_{L}}{s_{L}^{3}}=15 \nu \frac{\delta_{L}}{s_{L}^{3}}\left(\frac{u^{\prime}}{\lambda}\right)^{2}=15 \frac{\delta_{L}^{2}}{s_{L}^{3}} \frac{u^{\prime 2}}{\ell_{0}^{2}} \frac{\mathrm{Re}}{10} \\
& =\frac{3}{2} \frac{u^{\prime 3} \delta_{L}}{s_{L}^{3} \ell_{0}}=\frac{3}{2} \mathrm{Ka}^{2},
\end{aligned}
$$


using the scaling relations $\nu \sim \delta_{L} s_{L}$ (Williams 1985) and $\lambda \sim \ell_{0} \sqrt{10 / \operatorname{Re}}$ (Taylor 1935). With these substitutions in place, Eq. (2.5) simplifies to

$$
\frac{\Delta D}{D}=C^{\prime} \mathrm{Ka}^{\frac{2}{3}} \int_{1}^{\infty} \hat{\kappa}^{-11 / 6} e^{-\beta \hat{\kappa} /(2 \sqrt{\mathrm{Ka}})} d \hat{\kappa}
$$

where $C^{\prime} \equiv(2 C)^{\frac{1}{2}}\left(\frac{3}{2}\right)^{\frac{1}{3}} \sim 1$. This reflects a closed-form dependence of the enhancement in diffusivity due to small-scales $\eta<\ell<\delta_{L}$ on the Karlovitz number Ka. This is a novel result and provides an approach towards obtaining scaling relations for the diffusivity-related enhancement of $s_{T}$ in the TRZ regime. With the change of variable $t=\beta \hat{\kappa} /(2 \sqrt{\mathrm{Ka}})$, the integral is written as

$$
\frac{\Delta D}{D}=C^{\prime}\left(\frac{\beta}{2}\right)^{\frac{5}{6}} \mathrm{Ka}^{\frac{1}{4}} \int_{\beta /(2 \sqrt{\mathrm{Ka}})}^{\infty} t^{-11 / 6} e^{-t} d t
$$

which has the form of the upper incomplete gamma function $\Gamma(s, x)$ given by

$$
\Gamma(s, x) \equiv \int_{x}^{\infty} t^{s-1} e^{-t} d t=\Gamma(s)-\gamma(s, x),
$$

where $\Gamma(s)$ is the gamma function and $\gamma(s, x)$ is the lower incomplete gamma function, expanded in terms of the lower limit $x$ of the integral as

$$
\gamma(s, x)=x^{s} \sum_{k=0}^{\infty} \frac{(-x)^{k}}{k !(s+k)},
$$

where $s$ is the exponent in the integrand. Substituting the values $s=-5 / 6$ and $x=1$ in $\Gamma(s, x)$, the solution of Eq. (2.8) is written as

$$
\frac{\Delta D}{D}=C^{\prime}\left(\frac{\beta}{2}\right)^{\frac{5}{6}} \mathrm{Ka}^{\frac{1}{4}}\left[\Gamma\left(-\frac{5}{6}\right)-\gamma\left(-\frac{5}{6}, \frac{\beta}{2 \sqrt{\mathrm{Ka}}}\right)\right],
$$

which can be expanded in powers of Ka by applying Eq. (2.10) as follows

$$
\begin{aligned}
\frac{\Delta D}{D} & =C^{\prime}\left(\frac{\beta}{2}\right)^{\frac{5}{6}} \mathrm{Ka}^{\frac{1}{4}}\left[\Gamma\left(-\frac{5}{6}\right)-\sum_{k=0}^{\infty} \frac{(-1)^{k}(\beta / 2)^{k-5 / 6}}{k !\left(k-\frac{5}{6}\right)} \mathrm{Ka}\left(\frac{5}{12}-\frac{k}{2}\right)\right] \\
& =C^{\prime}\left[(6 / 5) \mathrm{Ka}^{\frac{2}{3}}+\Gamma(-5 / 6)(\beta / 2)^{5 / 6} \mathrm{Ka}^{\frac{1}{4}}+3 \beta \mathrm{Ka}^{\frac{1}{6}}-(3 / 28) \beta^{2} \mathrm{Ka}^{-\frac{1}{3}}+\ldots\right] \\
& =(6 / 5) C^{\prime} \mathrm{Ka}^{\frac{2}{3}}+\Gamma(-5 / 6)(\beta / 2)^{5 / 6} C^{\prime} \mathrm{Ka}^{\frac{1}{4}}+\mathcal{O}\left(\mathrm{Ka}^{\frac{1}{6}}\right)
\end{aligned}
$$

where $C^{\prime}$ and $\beta$ are empirically-derived constants. Therefore, the leading-order term for the overall enhancement in diffusivity scales as

$$
\frac{\Delta D}{D} \sim \mathrm{Ka}^{\frac{2}{3}}
$$

which leads to the scaling law $s_{T} \sim \mathrm{Ka}^{1 / 3}$ for the contribution of diffusivity enhancement towards $s_{T}$ enhancement for $\mathrm{Ka} \gg 1$. A similar form has been observed in recent DNS of thermonuclear flames in the small-scale turbulence regime (Aspden et al. 2010). For the range of $\mathrm{Ka}$ values $1<\mathrm{Ka}<1000$ considered here, Eq. (2.8) may also be evaluated numerically for each given value of Ka, thereby providing a profile of variation of the integral over a range of Ka. Regardless of the method of evaluation of Eq. (2.8), the enhancement in diffusivity due to small-scale turbulence $\ell<\delta_{L}$ is obtained as a closedform function of the Karlovitz number. 


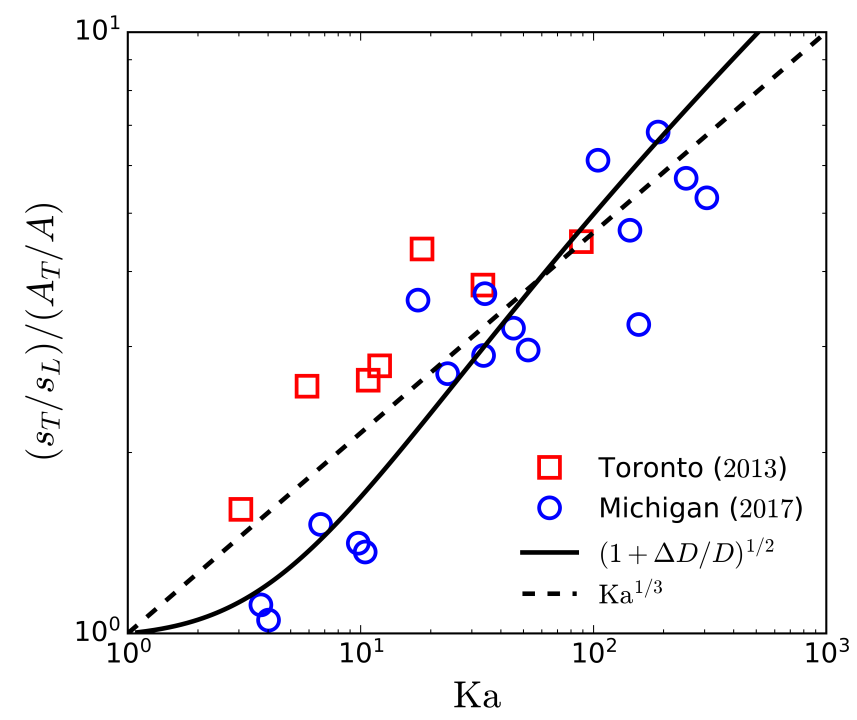

Figure 3. Measured disparity $\left(s_{T} / s_{L}\right) /\left(A_{T} / A\right)$ in the profiles of burning velocity enhancement and flame surface area enhancement as observed in the Toronto and Michigan experiments (symbols) and the enhancement factor (solid line) obtained from a numerical evaluation $I(\mathrm{Ka})$ of Eq. (2.8). Also shown is the leading-order term $\mathrm{Ka}^{1 / 3}$ (dashed line) obtained by solving Eq. (2.8) analytically using incomplete gamma functions.

\section{Result: burning velocity enhancement in small-scale turbulence}

The values of $\left(s_{T} / s_{L}\right) /\left(A_{T} / A\right)$ measured in Toronto and Michigan for a range of Karlovitz numbers are shown once again in Fig. 3. The contribution of diffusivity enhancement $D_{T} / D_{L}$ as estimated presently is shown alongside. Both the numerical evaluation $(1+\Delta D / D)^{1 / 2}$ of this contribution (solid line) and the analytically-obtained leading-order term $\mathrm{Ka}^{1 / 3}$ (dashed line) of the solution to Eq. (2.8) address, to a significant degree, the disparity in burning velocity enhancement $\left(s_{T} / s_{L}\right) /\left(A_{T} / A\right)$. On close examination, it is seen that the numerical evaluation approximates particularly well the profile of disparity measured in Michigan (blue circles in Fig. 3) for low Karlovitz numbers $1<\mathrm{Ka}<10$. The Toronto experiments (red squares in Fig. 3) fall mostly in the intermediate range of Karlovitz numbers $10<\mathrm{Ka}<100$ and show higher disparities than the Michigan experiments. Here, the leading-order term $\mathrm{Ka}^{1 / 3}$ appears to address the disparity better. For high Karlovitz numbers in the range $100<\mathrm{Ka}<1000$, both the leading-order term and the numerical evaluation predict similar disparities measured to be of the order $s_{T} / s_{L} \sim \mathcal{O}(10)$. Hence, the contribution of the enhancement factor $\Delta D / D$ towards the enhancement $s_{T} / s_{L}$ appears to be valid for the entire range of Karlovitz numbers in the measurements at Toronto and Michigan (Yuen \& Gülder 2013; Wabel et al. 2017).

The reconciliation of $\mathrm{DH} 1$ and $\mathrm{DH} 2$ provided by the present formulation suggests that DH2 may indeed operate alongside DH1 in the TRZ regime as hinted by previous investigations (Zimont 1979; Ronney \& Yakhot 1992; Gülder 2007; Lee \& Huh 2012). The operation of DH1 to enhance flame surface area is a consequence of turbulent length scales $\ell>\delta_{L}$, whereas the (simultaneous) operation of DH2 to enhance diffusivity is a consequence of turbulent length scales $\ell<\delta_{L}$. It is implicitly assumed that the enhancement due to DH1 does not interfere with the enhancement due to DH2, and that the operation of DH2 follows Eq. (1.3) for the entire range of $D_{T}$ values expected 
here. Such a simultaneous operation of DH1 and DH2 appears to address the measured overall enhancement of $s_{T}$ for high Ka values.

Estimation of the diffusive enhancement due to DH2 in terms of the Karlovitz number provides a means to reconcile existing measurements such as those considered here. Additional knowledge of the dependence of $\Delta A / A$ on relevant dimensionless groups such as the Karlovitz number could, in principle, aid the formulation of overall enhancement $s_{T} / s_{L}$ in terms of such dimensionless groups. Yet, it must be noted that the current reconciliation of $s_{T} / s_{L}$ with $A_{T} / A$ does not depend on flamelet quenching: $s_{T} / s_{L}$ as a function of $\mathrm{Ka}$ is formulated solely in terms of area enhancement of the flame surface and diffusivity enhancement in the flamelet preheat zone. While this is appropriate for addressing experimental data of Toronto (Gülder 2007) and Michigan (Wabel et al. 2017), further investigation is needed in order to address older experiments of Abdel-Gayed et al. (1987). DNS can provide necessary data to validate and improve the current formulation in this context. These ramifications warrant further investigation of the effective diffusivity enhancement in the Toronto and Michigan experiments with regard to a possible reconciliation between the different effects of Damköhler's first and second hypotheses (DH1 and DH2).

\section{Conclusions}

Measurements in turbulent Bunsen flames have long showed a discrepancy between the burning velocity enhancement $s_{T} / s_{L}$ and the flame surface area ratio $A_{T} / A$, which increases monotonically with the Karlovitz number Ka. It is shown that this discrepancy is commensurate with the effect of increase in diffusivity arising from the flux associated with turbulence small-scales $\ell<\delta_{L}$, according to Damköhler's second hypothesis (DH2). The diffusivity enhancement $D_{T} / D$ associated with these sub- $\delta_{L}$ scales is derived as a closed-form function of the Karlovitz number, which has implications with regard to sub-grid scale modelling of turbulent premixed flames. Notwithstanding the assumptions involved in the current estimation of $D_{T} / D$, the degree of reconciliation offered by the approach suggests that further experiments and models should investigate the possibility of a diffusive contribution from small-scale turbulence at high Ka values.

\section{Acknowledgements}

The authors thank Dr. Aaron Skiba for helping interpret the experimental data from Michigan. GVN thanks Dr. Sebastien Cespedes for all the fun discussions on the integral. GVN acknowledges the funding support of EPSRC grant EP/P022286/1.

\section{REFERENCES}

Abdel-Gayed, R. G., Bradley, D. \& Lawes, M. 1987 Turbulent burning velocities: a general correlation in terms of straining rates. Proceedings of the Royal Society A 414, 421-444.

Aspden, A., Woosley, S. \& Bell, J. B. 2010 Turbulence-flame interactions in type Ia supernovae. The Astrophysical Journal 689, 1173-1185.

Bradley, D. 1992 How fast can we burn? 24 ${ }^{\text {th }}$ Symposium (International) on Combustion pp. $247-262$.

Bray, K. N. C. \& Cant, R. S. 1991 Some applications of Kolmogorovs turbulence research in the field of combustion. Proceedings of the Royal Society A 434, 217-240.

DAmköHLeR, G. 1940 Der einfluss der turbulenz auf die flammengeschwindigkeit in gasgemschen. Zeitschrift für Elektrochemie und angewandte physikalische Chemie 46, 601652, (English translation: The effect of turbulence on the flame velocity in gas mixtures, NACA TM 1112, 1947). 
Driscoll, J. F. 2008 Turbulent premixed combustion: Flamelet structure and its effect on turbulent burning velocities. Progress in Combustion and Energy Science 34, 91-134.

GüLDER, Ö. 2007 Contribution of small scale turbulence to burning velocity of flamelets in the thin reaction zones regime. Proceedings of the Combustion Institute 31, 1369-1375.

von Karman, T. 1948 Progress in the statistical theory of turbulence. Proceedings of the National Academy of Sciences 34, 530-539.

Kraichnan, R. H. 1959 The structure of isotropic turbulence at very high Reynolds numbers. Journal of Fluid mechanics 5, 497-543.

LEe, D. \& HuH, K. Y. 2012 Validation of analytical expressions for turbulent burning velocity in stagnating and freely propagating turbulent premixed flames. Combustion and Flame 159, 1576-1591.

Lee, J., Lee, G. G. \& Huh, K. Y. 2014 Asymptotic expressions for turbulent burning velocity at the leading edge of a premixed flame brush and their validation by published measurement data. Physics of Fluids 26, 125103.

Peters, N. 1999 The turbulent burning velocity for large-scale and small-scale turbulence. Journal of Fluid mechanics 384, 107-132.

Pope, S. B. 2000 Turbulent flows. Cambridge University Press.

Ronney, P. D. \& YAкнот, V. 1992 Flame broadening effects on premixed turbulent flame speed. Combustion Science and Technology 86, 31-43.

ShepherD, I. G. 1996 Flame surface density and burning rate in premixed turbulent flames. Symposium (International) on Combustion 26, 373-379.

TAYlor, G. I. 1935 Statistical theory of turbulence. Proceedings of the Royal Society A 151, 421-444.

Veynante, D., Lodato, G., Domingo, P., Vervisch, L. \& Hawkes, E. R. 2010 Estimation of three-dimensional flame surface densities from planar images in turbulent premixed combustion. Experiments in Fluids 49, 267-278.

WABEL, T. M. 2017 An experimental investigation of premixed combustion in extreme turbulence. PhD thesis, University of Michigan (USA).

Wabel, T. M., Skiba, A. W. \& Driscoll, J. F. 2017 Turbulent burning velocity measurements: Extended to extreme levels of turbulence. Proceedings of the Combustion Institute 36, 1801-1808.

Wang, G-H., Clemens, N. T., Barlow, R. S. \& Varghese, P. L. 2007 A system model for assessing scalar dissipation measurement accuracy in turbulent flows. Measurement Science and Technology 18, 1287.

Wang, H., Hawkes, E. R. \& Chen, J. H. 2016 Turbulence-flame interactions in DNS of a laboratory high Karlovitz premixed turbulent jet flame. Physics of Fluids 28, 095107.

Williams, F. A. 1985 Combustion Theory, $3^{\text {rd }}$ edn. The Benjamin/Cummings Publishing Company, Inc.

Yuen, F. T. C. 2009 Experimental investigation of the dynamics and structure of lean-premixed turbulent combustion. PhD thesis, University of Toronto (Canada).

Yuen, F. T. C. \& GüLDER, Ö. 2013 Turbulent premixed flame front dynamics and implications for limits of flamelet hypothesis. Proceedings of the Combustion Institute 34, 1393-1400.

Zel'dovich, YA. B. \& Frank-KamenetskiĬ, D. A. 1938 Teorĭia teplovogo rasprostraneniia plameni. Zhurnal Fizicheskoi Khimii 12, 100-105, (English translation: A theory of flame propagation, In Selected Works of Yakov Borisovich Zeldovich, Volume I, Princeton University Press, 1992).

Zimont, V. L. 1979 Theory of turbulent combustion of a homogeneous fuel mixture at high Reynolds numbers. Combustion, Explosion and Shock Waves 15, 305-311. 